\title{
Variability, Genetic Diversity and Principal Component Analysis in Indian Mustard (Brassica juncea (L.) Czern. \& Coss.) for Seed Yield and Attributing Traits
}

\author{
Bhupendra Singh Yadav ${ }^{1 *}$, Hariom Kumar Sharma², \\ Ajay Pal Yadav and Bhagirath Ram $^{2}$ \\ ${ }^{1}$ MJRP University Jaipur Rajasthan, India \\ ${ }^{2}$ ICAR-Directorate of Rapeseed-Mustard Research, Bharatpur, Rajasthan, India \\ *Corresponding author
}

\section{A B S T R A C T}

\section{Keywords}

Brassica juncea, Genetic variability, Genetic advance, Heritability,

Principal component analysis

Article Info

\section{Accepted:}

15 January 2021

Available Online:

10 February 2021
Fifty genotypes of Indian mustard [Brassica juncea (L.) Czern.\& Coss.] were evaluated for fourteen quantitative traits. The experiment was conducted at research of farm, ICAR-Directorate of Rapeseed-Mustard Research, Sewar, Bharatpur, (Rajasthan) during rabi 2019-20. Both genotypic and phenotypic coefficients of variation were higher for important traits including main shoot length, days to maturity, seed yield per plant, thousand seed weight. High heritability in conjunction with high genetic advance were observed for seed yield per plant, thousand seed weight, fruiting zone length, days to initial flowering, first basal branch, siliqua length, seeds per siliqua, primary branches, main shoot length, days to maturity, secondary branch, plant height, siliqua on main shoot, days to $50 \%$ flowering. The fifty germplasm were grouped in seven clusters. Cluster 1 was the largest group (12 germplasm) followed by cluster 2 (11 germplasm), cluster 5 ( 9 germplasm), cluster 4 (8 germplasm) and cluster 3 (7 germplasm), cluster 7 ( 2 germplasm) and cluster 6 is the smallest having only one germplasm (Table 3 and Fig 1). The Principal component analysis (PCA) was conducted using quantitative traits. First PC had 262.479 variance (eigen value) which is $67.08 \%$ of total variation explained (Table 3), followed by PCII $(10.48 \%)$ and PC-III (8.35\%), PC-IV (4.78\%), PC-V (4.55\%). Cumulatively first five components explained $95.25 \%$ of the total variation in the data.

\section{Introduction}

Indian Mustard (Brassica juncea L. czern.\& coss. $2 \mathrm{n}=36$, AABB genome), is an allopolyploid brassica species. It contributes more than $80 \%$ to the total rapeseed-mustard production in the country and is an important component in the oilseed sector. It is known to be more drought tolerant and shattering resistant than B. napus and B. rapa, therefore, has an enormous cultivation potential in semiarid areas. It is mainly self-pollinating species, 
despite that an average of 7.5 to 30 percent out-crossing does occur under natural field conditions. Mustard is a cool season crop, which requires temperature range from 6 to $27^{\circ} \mathrm{C}$, mustard follows C3 pathway for carbon assimilation. Mustard is generally grown under rainfed conditions. It requires well drained soil having $\mathrm{pH}$ near to neutral. It has a low water requirement $(240-400 \mathrm{~mm})$ which fits well in the rainfed cropping system. Nearly $20 \%$ area of mustard is under rainfed condition.

Rapeseed-mustard accounts for nearly onethird of the oil produced in India, making it the country's key edible oilseed crop. Rapseed and mustard sown in 5.96 million hectare and production was 8.32 million tonnes in 201718. Rajasthan is one of the major mustard producing states in the country, contributing $40.87 \%$ of total production of India. National and state yield of mustard in 2017-18 are 1397 $\mathrm{kg} / \mathrm{ha}$ and $1558 \mathrm{~kg} / \mathrm{ha}$, respectively (Agricultural statistics at a glance 2018) Although, yield of mustard in Rajasthan is more than its national average yield.

Among the various constraints attributing to low productivity of mustard in arid and semiarid region, the erratic nature of climate, inefficient irrigation water, biotic stresses (white rust, stem rot, aphids, painted but) and abiotic stresses (high temperature at sowing time), fertilizer management and poor soil physical conditions are the most important factors which lead to the low crop yield.

Evaluation of genetic divergence and relatedness among breeding materials has significant implications for the improvement of crop plants. Furthermore, genetic diversity could help breeders and geneticists to understand the structure of germplasm, predict which combinations would produce the best offsprings (Hu et al., 2007), and facilitate to widen the genetic basis of breeding material for selection (Qi et al., 2008). Genetic diversity among individuals or populations can be determined using morphological, biochemical and molecular approaches (Mohammadi and Prasanna 2003). Assessment of genetic diversity in $B$. juncea using phenotypic characters has previously been done by many researchers (Vaishnava et al., 2006, Alie et al., 2009, Singh et al., 2010).

\section{Materials and Methods}

The materials for the present investigation consisted of 50 genotypes of Indian mustard obtained from germplasm unit of ICARDirectorate of Rapeseed-Mustard Research, Sewar, Bharatpur. 50 genotypes of Indian mustard including 5 check varieties (NPJ-112, Pusa bold, PM-26, CS-56, Kranti) were evaluated in RCBD with two replications under irrigated timely sown condition. Sowing was done on 15-10-2019.

In each replication each genotype was sown in a plot of one row of 3-meter length. Plant to plant spacing was maintained at $10 \mathrm{~cm}$ by thinning after 15-20 days of sowing. Observation Recorded five competitive plants were randomly selected at the time of maturity (except the days to 50 per cent flowering and days to maturity which were recorded on plot basis) Plant height $(\mathrm{cm})$, Primary branches per plant, Secondary branches per plant, Main shoot length $(\mathrm{cm})$, Siliqua on main shoot, Fruiting zone length $(\mathrm{cm})$, First basal branching $(\mathrm{cm})$, Initial flowering, Days to 50 per cent flowering, Days to maturity, Siliqua length $(\mathrm{cm})$, Seeds per siliqua, Seed yield per plant (g), Thousand seed weight (g). The phenotypic and genotypic coefficients of variation (GCV and PCV), heritability in broad sense, genetic advance as \% of mean, correlation coefficients at genotypic and phenotypic level and principal component analysis computed using standard statistical methods. 


\section{Results and Discussion}

The analysis of variance was carried out for twelve characters and showing the significant difference amongst all the parents except biological yield, among the F1's except number of secondary branches per plant, no. of seed per siliqua and biological yield per plant, parents vs F1's for all the characters revealed significant difference (Patel et al., 2012; Arifullah, 2013). Highly significant differences were recorded among the treatments for all the characters namely fruiting zone length, first basal branch, initial flowering, siliqua length, and significant differences were observed for the characters plant height, primary branches per plant, secondary branches per plant, main shoot length, siliqua on main shoot, days to $50 \%$ flowering, days to maturity, seeds per siliqua, thousand seed weight (Table 1 and 2).

Estimates of PCV and GCV were observed that phenotypic variance were higher than the corresponding genotypic variance for all the traits studies. Data presented in (Table 2) showed maximum GCV and PCV was recorded for First basal branch, Seed yield/ plant (g), Thousand seed weight, Fruiting zone length, Seeds/ siliqua. High heritability in conjunction with high genetic advance were observed for seed yield per plant, thousand seed weight, fruiting zone length, days to initial flowering, first basal branch, siliqua length, seeds per siliqua, primary branch, main shoot length, days to maturity, secondary branch, plant height, siliqua on main shoot, days to $50 \%$ flowering,

\section{Multivariate analysis}

\section{Principal component analysis}

The PCA was conducted using 14 quantitative traits in Indian mustard and presented in table 3. First PC had 262.479 variance (eigen value) which is $67.08 \%$ of total variation explained
(Table 3), followed by PC-II (10.48\%) and PC-III (8.35\%), PC-IV (4.78\%), PC-V (4.55\%). Cumulatively five six components explained $95.25 \%$ of the total variation in the data.

PC-I had significantly positive correlation $(\mathrm{P}<$ 0.30 ) with five variables (plant height, fruiting zone length, first basal branching, days to initial flowering, days to $50 \%$ flowering). PCII had positive correlation $(\mathrm{P}<0.05)$ with two variables (secondary branches, seed yield/plant) whereas, PC-III had positive correlation $(\mathrm{P}<0.05)$ with three variables (plant height, silique on main shoot, seed yield/plant). Similar results have also been reported earlier by Zada et al., (2013) in Ethiopian mustard; Avtar et al., (2014) in toria and Neeru et al., (2015) in Indian mustard.

\section{Genetic diversity based on Hierarchical cluster analysis}

The random clustering pattern of genotypes i.e. grouping of genotypes from different regions in the same cluster (three local genotypes CAULC-2, CAULC-3, CAULC-4 from Manipur were grouped together with Kranti from Pantnagar and PM-28 from New Delhi) indicated that the genetic diversity of the genotypes is not necessarily related with the distribution of genotypes in different parts of the country, as supported by earlier findings of Jeena and Sheikh (2003), Pandey et al., (2013), Dar et al., (2010) and Gupta et al., (2015).

The genetic diversity, among the genotypes in the present study may be resulted from genetic drift and selection that cause greater diversity than geographical distribution as suggested by Murty and Arunachalam (1966). Based on usual Euclidean distance, fifty genotypes of Indian mustard were grouped into seven major clusters. Dendrogram was constructed based on 14 quantitative traits using DARwin 6 software (Fig. 1). 
Table.1 Analysis of variance (ANOVA) for seed yield and yield attributing characters in Indian mustard

\begin{tabular}{|c|c|c|c|c|c|c|c|c|c|c|c|c|c|c|c|}
\hline \multirow[b]{2}{*}{$\begin{array}{l}\text { Source of } \\
\text { variation }\end{array}$} & \multirow[b]{2}{*}{$\begin{array}{c}\text { Degree } \\
\text { of } \\
\text { freedom }\end{array}$} & \multicolumn{14}{|c|}{ Mean Square } \\
\hline & & $\begin{array}{l}\text { Plant } \\
\text { height }\end{array}$ & $\begin{array}{l}\text { Primary } \\
\text { branch }\end{array}$ & $\begin{array}{c}\text { Second } \\
\text { ary } \\
\text { branch }\end{array}$ & $\begin{array}{c}\text { Main } \\
\text { shoot } \\
\text { length }\end{array}$ & $\begin{array}{c}\text { Siliqua on } \\
\text { main } \\
\text { shoot }\end{array}$ & $\begin{array}{l}\text { Fruiting } \\
\text { zone } \\
\text { length }\end{array}$ & $\begin{array}{c}\text { First basal } \\
\text { branch }\end{array}$ & $\begin{array}{c}\text { Days to } \\
\text { initial } \\
\text { flowering }\end{array}$ & $\begin{array}{c}\text { Days to } \\
50 \% \\
\text { flowering }\end{array}$ & $\begin{array}{l}\text { Days to } \\
\text { maturing } \\
\text { maturity }\end{array}$ & $\begin{array}{c}\text { Seed } \\
\text { yield per } \\
\text { plant }\end{array}$ & $\begin{array}{l}\text { Siliqua } \\
\text { Length }\end{array}$ & $\begin{array}{l}\text { Seeds } \\
\text { per } \\
\text { siliqua }\end{array}$ & $\begin{array}{c}\text { Thousa } \\
\text { nd seed } \\
\text { weight }\end{array}$ \\
\hline Replication & 1 & 210.25 & 2.49 & 27.25 & 19.15 & 0.66 & 136 & 30.25 & 16 & 42.26 & 15.22 & 14.82 & 0.98 & 1.44 & 0.89 \\
\hline Treatment & 49 & $10321.75 *$ & $0.760 *$ & $4.79 *$ & $20.76 *$ & $9.52 *$ & $350.69 * *$ & $97.77 * *$ & $29.58 * *$ & $14.44 *$ & $6.55 *$ & $30.05 * *$ & $0.16 * *$ & $3.39 *$ & $0.84 *$ \\
\hline Error & 99 & 5709.25 & .403 & 2.58 & 11.05 & 5.28 & 98.07 & 14.98 & 10.77 & 8.90 & 3.51 & 3.68 & 0.80 & 1.78 & 0.21 \\
\hline SEM ( \pm$)$ & & 7.632 & 0.449 & 1.137 & 2.35 & 1.625 & 7.002 & 2.73 & 2.321 & 2.109 & 1.325 & 1.35 & 0.2 & 0.945 & 0.33 \\
\hline CV (\%) & & 5.854 & 8.673 & 9.714 & 5.328 & 5.004 & 10.193 & 14.14 & 6.893 & 5.296 & 1.366 & 11.78 & 7.354 & 11.871 & 11.85 \\
\hline CD (1\%) & & 28.929 & 1.702 & 4.31 & 8.909 & 6.162 & 26.541 & 10.37 & 8.797 & 7.996 & 5.025 & 5.14 & 0.761 & 3.582 & 1.25 \\
\hline CD (5\%) & & 21.692 & 1.276 & 3.231 & 6.68 & 4.62 & 19.901 & 7.77 & 6.596 & 5.996 & 3.768 & 3.85 & 0.57 & 2.686 & 0.93 \\
\hline
\end{tabular}

Table.2 Estimates of genotypic (GCV) and phenotypic (PCV) co-efficient of variation, heritability (bs) and genetic advance (\% of mean) for seed yield and component traits in Indian mustard.

\begin{tabular}{|c|c|c|c|c|c|c|c|}
\hline \multirow[t]{2}{*}{ Characters } & \multirow[t]{2}{*}{ Mean } & \multicolumn{2}{|c|}{ Range } & \multirow[t]{2}{*}{ GCV } & \multirow[t]{2}{*}{ PCV } & \multirow[t]{2}{*}{ Heritability \% } & \multirow{2}{*}{$\begin{array}{c}\text { Genetic advance (\% } \\
\text { of means) }\end{array}$} \\
\hline & & min. & max. & & & & \\
\hline Plant height & 184.37 & 166 & 210 & 3.721 & 6.937 & 28.773 & 4.112 \\
\hline Primary branch & 7.32 & 6.2 & 8.9 & 5.769 & 10.417 & 30.673 & 6.582 \\
\hline Secondary branch & 16.55 & 12.9 & 20.8 & 6.349 & 11.605 & 29.924 & 7.154 \\
\hline Main shoot length & 62.38 & 57.5 & 73 & 3.534 & 6.394 & 30.543 & 4.023 \\
\hline Siliqua on main shoot & 45.94 & 41.5 & 50 & 3.169 & 5.924 & 28.622 & 3.493 \\
\hline Fruiting zone length & 97.15 & 67.5 & 146 & 11.569 & 15.419 & 56.292 & 17.88 \\
\hline First basal branch & 27.37 & 16.5 & 47.5 & 18.325 & 31.135 & 34.641 & 22.218 \\
\hline Days Initial flowering & 47.62 & 42 & 57 & 6.44 & 9.433 & 46.601 & 9.056 \\
\hline Days to $50 \%$ flowering & 56.33 & 52 & 61 & 2.954 & 6.065 & 23.723 & 2.964 \\
\hline Days to maturity & 137.21 & 132 & 140 & 0.898 & 1.635 & 30.159 & 1.016 \\
\hline Seed yield/ plant (g) & 16.27 & 8.8 & 24.7 & 22.31 & 25.233 & 78.172 & 40.634 \\
\hline Siliqua length & 3.86 & 3.3 & 4.5 & 5.272 & 9.049 & 33.941 & 6.327 \\
\hline Seeds/ siliqua & 11.26 & 8 & 13 & 7.958 & 14.292 & 31.001 & 9.127 \\
\hline Thousand seed weight & 3.93 & 2.38 & 5.05 & 14.212 & 18.507 & 58.967 & 22.481 \\
\hline
\end{tabular}


Table.3 Eigen vectors, eigen values and proportion of variation explained by different components for different quantitative traits of Indian mustard

\begin{tabular}{|c|c|c|c|c|c|}
\hline Trait & PC 1 & PC 2 & PC 3 & PC 4 & PC 5 \\
\hline Plant height & 0.561 & 0.439 & 0.637 & -0.283 & -0.001 \\
\hline Primary branch & 0.001 & 0.028 & 0.012 & 0.021 & 0.051 \\
\hline Secondary branch & -0.006 & 0.112 & 0.004 & 0.085 & 0.106 \\
\hline Main shoot length & 0.028 & 0.076 & 0.053 & 0.489 & 0.081 \\
\hline Siliqua on main shoot & 0.003 & 0.093 & 0.084 & 0.291 & -0.030 \\
\hline Fruiting zone length & 0.789 & -0.098 & -0.576 & 0.100 & -0.143 \\
\hline First basal branch & 0.216 & -0.849 & 0.454 & 0.123 & 0.041 \\
\hline Days Initial flowering & 0.105 & 0.044 & -0.056 & 0.129 & 0.779 \\
\hline Days to 50 \% flowering & 0.048 & 0.020 & -0.043 & 0.009 & 0.558 \\
\hline Days to maturity & -0.009 & 0.032 & 0.064 & 0.052 & -0.068 \\
\hline Seed yield/ plant (g) & 0.034 & 0.207 & 0.190 & 0.730 & -0.174 \\
\hline Siliqua length & 0.003 & -0.005 & 0.002 & -0.003 & 0.007 \\
\hline Seeds/ siliqua & 0.007 & 0.051 & -0.030 & 0.100 & -0.054 \\
\hline Thousand seed weight & 0.007 & 0.002 & 0.007 & 0.026 & -0.026 \\
\hline Eigenvalue & 262.479 & 41.011 & 32.678 & 18.708 & 17.828 \\
\hline \% variance & 67.085 & 10.482 & 8.352 & 4.782 & 4.556 \\
\hline Cummulative variance & 67.085 & 77.567 & 85.919 & 90.701 & 95.257 \\
\hline
\end{tabular}


Table.4 Cluster mean for different quantitative traits of Indian mustard

\begin{tabular}{|c|c|c|c|c|c|c|c|c|c|c|c|c|c|c|}
\hline Clusters & $\begin{array}{c}\text { Plant } \\
\text { height }\end{array}$ & $\begin{array}{l}\text { Primary } \\
\text { branch }\end{array}$ & $\begin{array}{l}\text { Secondary } \\
\text { branch }\end{array}$ & $\begin{array}{l}\text { Main } \\
\text { shoot } \\
\text { length }\end{array}$ & $\begin{array}{l}\text { Siliqua on } \\
\text { main shoot }\end{array}$ & $\begin{array}{l}\text { Fruiting } \\
\text { zone } \\
\text { length }\end{array}$ & $\begin{array}{c}\text { First } \\
\text { basal } \\
\text { branch }\end{array}$ & $\begin{array}{c}\text { Days to } \\
\text { initial } \\
\text { flowering }\end{array}$ & $\begin{array}{c}\text { Days } \\
50 \% \\
\text { flowering }\end{array}$ & $\begin{array}{l}\text { Days to } \\
\text { maturity }\end{array}$ & $\begin{array}{l}\text { Seed } \\
\text { yield/ } \\
\text { plant }\end{array}$ & $\begin{array}{l}\text { Siliqua } \\
\text { length }\end{array}$ & $\begin{array}{l}\text { Seeds/ } \\
\text { siliqua }\end{array}$ & $\begin{array}{c}\text { Thousand } \\
\text { Seed } \\
\text { Weight }\end{array}$ \\
\hline 1 & 187.87 & 7.54 & 16.95 & 62.96 & 45.35 & 99.08 & 26.16 & 50.08 & 57.33 & 137.45 & 15.89 & 3.87 & 11.29 & 3.65 \\
\hline 2 & 184.4 & 7.29 & 16.5 & 61.45 & 45.79 & 89 & 23.13 & 44.72 & 55.27 & 138.36 & 16.48 & 3.75 & 11.31 & 3.77 \\
\hline 3 & 180 & 7.11 & 15.7 & 63.64 & 46.18 & 92.14 & 35.07 & 46.35 & 55.42 & 136.64 & 17.44 & 3.8 & 10.71 & 4 \\
\hline 4 & 170.62 & 7.38 & 17.1 & 62.31 & 46.31 & 87 & 22.56 & 47.18 & 56 & 136.75 & 14.76 & 3.85 & 11.12 & 3.84 \\
\hline 5 & 194.5 & 7.02 & 15.91 & 62.05 & 46.44 & 111.77 & 32.88 & 48.94 & 56.94 & 136.11 & 16.93 & 3.99 & 11.16 & 4 \\
\hline 6 & 154 & 7.3 & 17.6 & 58.5 & 45.9 & 67.5 & 16.5 & 42.5 & 54.5 & 137.5 & 8.8 & 3.8 & 10 & 2.725 \\
\hline 7 & 203 & 8 & 17.55 & 63.25 & 45.8 & 137.5 & 30.75 & 51.5 & 58.75 & 138 & 14.9 & 3.77 & 11.75 & 4.07 \\
\hline
\end{tabular}

Table.5 Distribution pattern of 50 genotypes of Indian mustard into seven cluster based on Euclidean distance

\begin{tabular}{|c|c|c|c|c|}
\hline Cluster & Genotype & No. of genotypes & $\begin{array}{l}\text { Best performing genotype } \\
\text { for seed yield/plant (g) }\end{array}$ & $\begin{array}{l}\text { Best performing genotype } \\
\text { for } 1000 \text {-seed weight (g) }\end{array}$ \\
\hline 1 & $\begin{array}{l}\text { DRMR 1179, DRMR 439, DRMR 85, DRMR 437, } \\
\text { DRMR 1157, DRMR 443, DRMR 1171, DRMR 564, } \\
\text { DRMR 1431, DRMR 468, DRMR 80, NPJ } 112\end{array}$ & 12 & DRMR $468(24.7)$ & DRMR $1431(4.735)$ \\
\hline 2 & $\begin{array}{l}\text { DRMR 60, DRMR 1147, DRMR 1105,DRMR 1275, } \\
\text { DRMR 556, DRMR 1434, DRMR 120, DRMR 69, } \\
\text { DRMR 1188, BPR 541-4, DRMR } 1148\end{array}$ & 11 & DRMR 1148 (22.6) & DRMR 1188 (4.58) \\
\hline 3 & $\begin{array}{c}\text { DRMR 59, DRMR 1190, DRMR 466, DRMR 74, DRMR } \\
\text { 1208, DRMR 1639, DRMR } 473\end{array}$ & 7 & DRMR 59 (24.4) & DRMR 466 (4.58) \\
\hline 4 & $\begin{array}{l}\text { DRMR 1146, DRMR 569, DRMR 1187, DRMR 1144, } \\
\text { DRMR 567, DRMR 423, DRMR 1192, DRMR } 1210\end{array}$ & 8 & DRMR 1192 (24.3) & DRMR 1144 (4.84) \\
\hline 5 & $\begin{array}{l}\text { DRMR 1153, DRMR 389, DRMR 1167, Kranti, Pusa } \\
\text { Bold, DRMR 474, DRMR 1380, DRMR 1347, DRMR } 84\end{array}$ & 9 & KRANTI (21.7) & PUSA BOLD (4.98) \\
\hline 6 & PM 26 & 1 & PM $26(8.8)$ & PM 26 (2.72) \\
\hline 7 & CS 56, DRMR 1154 & 2 & CS $56(17.2)$ & CS 56 (4.69) \\
\hline
\end{tabular}


Fig.1 UPGMA dendrogram based on Euclidean hierarchical clustering

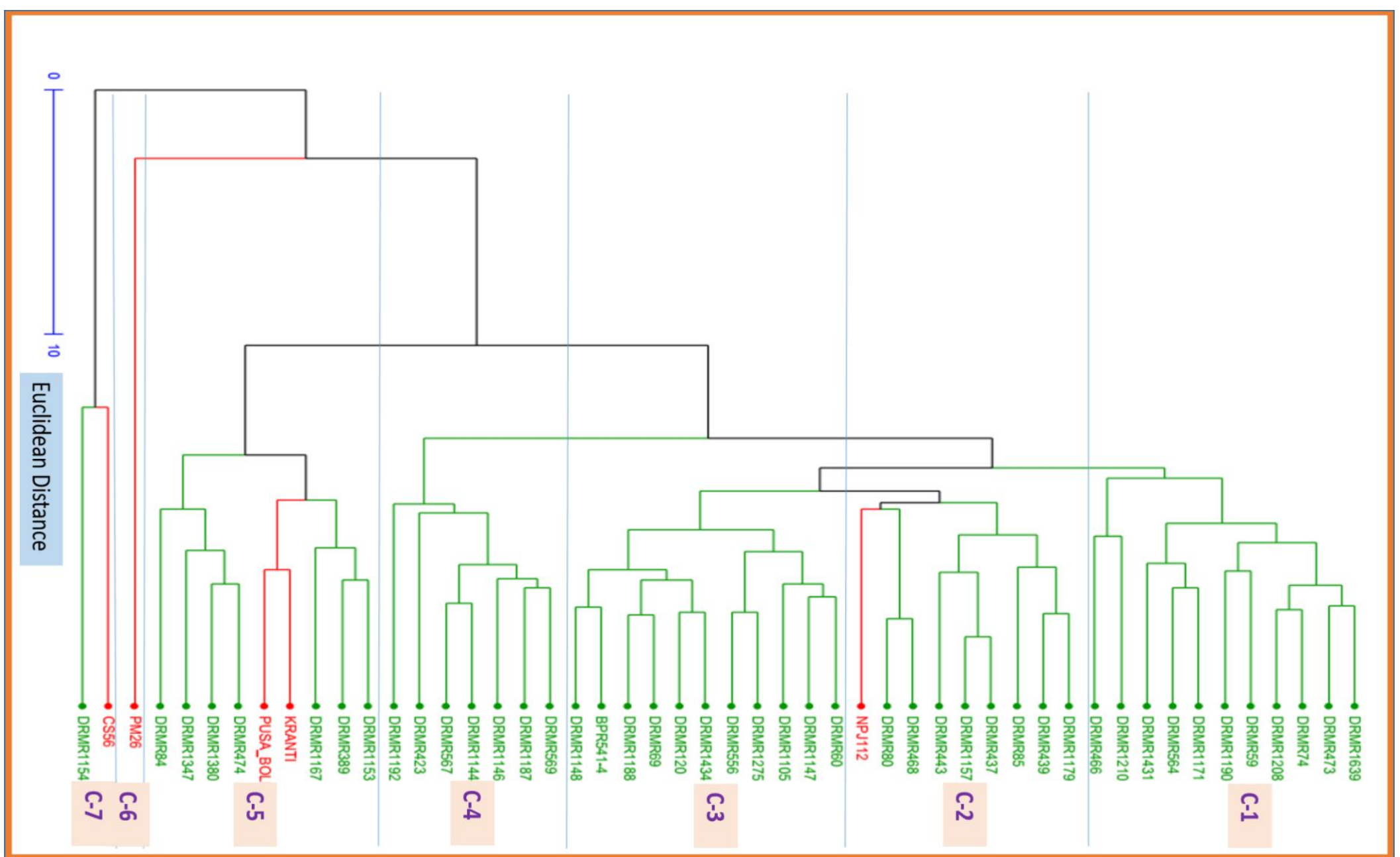


Cluster 1 was the largest group (12 genotypes; DRMR 1179, DRMR 439, DRMR 85, DRMR 437, DRMR 1157, DRMR 443, DRMR 1171, DRMR 564, DRMR 1431, DRMR 468, DRMR 80, NPJ 112), followed by cluster 2 (11 genotypes; DRMR 60, DRMR 1147, DRMR 1105,DRMR 1275, DRMR 556, DRMR 1434, DRMR 120, DRMR 69, DRMR 1188, BPR 541-4, DRMR 1148), cluster 5 (9 genotypes; DRMR 1153, DRMR 389, DRMR 1167, Kranti, Pusa Bold, DRMR 474, DRMR 1380, DRMR 1347, DRMR 84), cluster 4 (8 genotypes; DRMR 1146, DRMR 569, DRMR 1187, DRMR 1144, DRMR 567, DRMR 423, DRMR 1192, DRMR 1210) and cluster 3 (7 genotypes; DRMR 59, DRMR 1190, DRMR 466, DRMR 74, DRMR 1208, DRMR 1639, DRMR 473), cluster 7 (2 genotypes; CS 56, DRMR 1154) and cluster 6 is the smallest having only one genotype (PM 26) (Table 4).

The cluster mean of fourteen different characters are being presented in Table 4 . Cluster 3 showed high cluster mean for seed yield $(17.4 \mathrm{~g})$, while cluster $3,5,7$ showed high cluster mean for 1000-seed weight $(>4.0 \mathrm{~g})$.

As far as best performing line is concern, for cluster 1 DRMR 468 showed high seed yield/plant $(24.7 \mathrm{~g})$. In cluster 3, DRMR 59 is best performing genotype $(24.4 \mathrm{~g})$ while in cluster 4, DRMR 1192 recorded highest yield (24.3g) (Table 4).

For 1000-seed weight 5.05g (DRMR473) exhibited highest in cluster 3. Genotypes in same cluster are genetically more similar as compared to genotypes in other clusters. Hence, to get more seed yield/plant genotypes of cluster 3 can be crossed with genotypes of cluster five. Best performing lines from each cluster can be used in hybridization programme to generate more variability for different traits of interest.

\section{References}

Agricultural Statistics at a glance. 2018. Government of India. 110-112.

Alie F.A., Singh T., Tariq., Sharma P.K. 2009. Genetic diversity analysis in Indian mustard [Brassica juncea (L.) Czern \& Coss]. Progressive Agriculture-An International Journal. 9:50-53.

Arifullah M., Munir M., Mahmood A., Ajmal K.S., Hassanul- F. 2013. Genetic analysis of some yield attributes in Indian mustard (B. juncea L.). Afri $J P l$ Sci.7:219-226.

Avtar R., Singh D., Thakral N.K., Singh A., Sangwan O., Rani B., Kumari N. 2014. Multivariate analysis for evaluation and classification of toria germplasm accessions. Res. Crops 15(1):129-134.

Dar Z.A., Wani S.A., Zaffar G., Ishfaq A., Wani M.A., Habib M., Khan M.H., Razvi S.M. 2010. Genetic divergence studies in brown sarson (Brassica rapa L.). Res. J. Agric. Sci., 1(2):140-141.

Gupta R.K., Dwivedi V.K. Meena B. 2015. Genetic divergence analysis for seed yield parameters and oil content in Indian mustard (Brassica juncea L. Czern and Coss). Indian Res. J. Genet. Biotech., 7(1):84-86.

Hu S., Yu C., Zhao H., Sun G., Zhao S., Vyvadilova M.et al., 2007. Genetic diversity of (Brassica napus L.) Germplasm from China and Europe assessed by some agronomically important characters. Euphytica. 154:9-16.

Jeena A.S., Sheikh F.A. 2003. Genetic divergence analysis in gobhi sarson (Brassica napus L). J. Oilseed Res. 20:210-212.

Mohammadi S.A., Prasanna B.M. 2003. Analysis of genetic diversity in crop plants salient statistical tools and considerations. Review and 
Interpretation. Crop Science. 43:12351248.

Murty B.R., Arunachalam V. 1966. The nature of genetic divergence in relation to breeding system in some crop plants. Indian J. Genet. 26:188-198.

Neeru., Thakral N.K., Avtar R., Singh A. 2015. Evaluation and classification of Indian mustard (Brassica juncea L.) genotypes using principal component analysis. J. Oilseed Brassica.6(1):167174.

Pandey R., Kumar B., Kumar M. 2013. Genetic divergence for quantitative traits in Indian mustard (Brassica juncea L. Czern. and Coss). AmericanEurasian J. Agric. Environ. Sci., 13(3): 348-351.

Patel A.M., Prajapati D.B., Patel D.G. 2012. Heterosis and combining ability studies in Indian mustard (B. juncea L.). Ind J Sci Res Tech., 1:38-40

Qi X., Yang J., Zhang M. 2008. AFLP-based genetic diversity assessment among Chinese vegetable mustards (Brassica juncea (L.) Czern. \& Coss.). Genetic Resources and Crop Evolution. 55:705-711.

Singh Dhiraj., Arya Rajesh Kumar., Chandra Navin., Niwas Ram., Salisbury Phillip. 2010. Genetic diversity studies in relation to seed yield and its component traits in Indian mustard (Brassica juncea L. Czern \& Coss.). Journal of Oilseed Brassica. 1(1):1922.

Vaishnava A., Sachan J.N., Tewari S.K. 2006. Genetic divergence for important quantitative traits in Indian mustard (Brassica juncea (L.) Czern and Coss). Agricultural Science Digest. 26:269272.

Zada M., Zakir N., Rabbani M., Shinwari A., Khan Z. 2013. Assessment of genetic diversity in Ethiopian mustard (Brassica carinata A. Brun) germplasm using multivariate techniques. Pak. J. Bot. 45(SI): 583593.

\section{How to cite this article:}

Bhupendra Singh Yadav, Hariom Kumar Sharma, Ajay Pal Yadav and Bhagirath Ram. 2021. Variability, Genetic Diversity and Principal Component Analysis in Indian Mustard (Brassica juncea (L.) Czern.\& Coss.) for Seed Yield and Attributing Traits. Int.J.Curr.Microbiol.App.Sci. 10(02): 1769-1777. doi: https://doi.org/10.20546/ijcmas.2021.1002.209 